\title{
Probe-based association analysis identifies several deletions associated with average daily gain in beef cattle
}

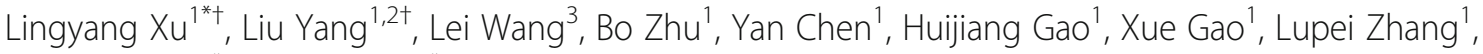
George E. Liu ${ }^{4^{*}}$ and Junya Li $i^{i^{*}}$

\begin{abstract}
Background: Average daily gain (ADG) is an important trait that contributes to the production efficiency and economic benefits in the beef cattle industry. The molecular mechanisms of ADG have not yet been fully explored because most recent association studies for ADG are based on SNPs or haplotypes. We reported a systematic CNV discovery and association analysis for ADG in Chinese Simmental beef cattle.

Results: Our study identified 4912 nonredundant CNVRs with a total length of $\sim 248.7 \mathrm{Mb}$, corresponding to $\sim 8.9 \%$ of the cattle genome. Using probe-based CNV association, we identified 24 and 12 significant SNP probes within five deletions and two duplications for ADG, respectively. Among them, we found one common deletion with $89 \mathrm{~kb}$ imbedded in LHFPL Tetraspan Subfamily Member 6 (LHFPL6) at $22.9 \mathrm{Mb}$ on BTA12, which has high frequency (12.9\%) dispersing across population. CNV selection test using $V_{S T}$ statistic suggested this common deletion may be under positive selection in Chinese Simmental cattle. Moreover, this deletion was not overlapped with any candidate SNP for ADG compared with previous SNPs-based association studies, suggesting its important role for ADG. In addition, we identified one rare deletion near gene Growth Factor Receptor-bound Protein 10 (GRB10) at 5.1 Mb on BTA4 for ADG using both probe-based association and region-based approaches.
\end{abstract}

Conclusions: Our results provided some valuable insights to elucidate the genetic basis of ADG in beef cattle, and these findings offer an alternative perspective to understand the genetic mechanism of complex traits in terms of copy number variations in farm animals.

Keywords: Copy number variation, Average daily gain, Probe-based association, Positive selection, Beef cattle

\section{Background}

Genomic structural variants mainly comprised of copy number variations (CNVs) in the form of large-scale insertions and deletions, as well as inversions and translocations [1]. CNVs involve more genomic sequence as compared to nucleotide polymorphisms (SNPs), thus they have potentially larger effects, including alternating gene regulation and dosage, contributing to gene expression and risk for normal phenotypic variability [2-5].

\footnotetext{
*Correspondence: xulingyang@163.com; george.liu@ars.usda.gov; j11@iascaas.net.cn

${ }^{+}$Lingyang Xu and Liu Yang contributed equally to this work.

'Innovation Team of Cattle Genetic Breeding, Institute of Animal Sciences,

Chinese Academy of Agricultural Sciences, Beijing 100193, China

${ }^{4}$ U.S. Department of Agriculture-Agricultural Research Services, Animal

Genomics and Improvement Laboratory, Beltsville, MD 20705, USA

Full list of author information is available at the end of the article
}

High-throughput SNP genotyping arrays have been widely used in genome-wide studies. While these arrays have limited capacity to assess the effects of rare single-site variants, they can be readily used to identify large copy number variations, even if they occur in only a few subjects [6]. There are tremendous evidences showing that other genetic variants like copy number variations may affect complex traits, including short stature and anthropometric traits in human $[7,8]$. For instance, one recent study suggested that a $45 \mathrm{~kb}$ deletion was associated with the body mass index in humans, which also reflects neuronal influence of the deletion on body weight regulation [9]. Previous study identified several genes (e.g., MC4R, FIBIN, and FMO5), harboring both common and rare variants which may affect body 
size and anthropometric traits using a CNV-association analysis in European adults [8].

Considerable attention has turned towards assessing the association between copy number variations and complex traits in farm animals using high-throughput array. In cattle, several studies have found CNVs are likely to be associated with resistance to gastrointestinal nematodes in Angus [10,11] and residual feed intake, milk production and fertility traits in Holstein cows [1214]. Also, a recent study described a $660 \mathrm{~kb}$ deletion which has antagonistic effects on fertility and milk production in Nordic Red cattle [15]. Thus, detecting CNVs and identifying their potential associations have gradually become an alternative method to comprehensively elucidate the genetic mechanism of complex traits in farm animals.

Average daily gain (ADG) is generally recognized as an economically important growth trait that contributes to the production benefits in the beef industry. Previous studies have identified many QTL regions associated with ADG in various populations [16-25], these studies had utilized multiple methods including SNP-based GWAS, haplotype-based GWAS and gene-based GWAS to test the association for ADG in various populations. However, the molecular mechanism of ADG have not yet been fully explored, partially because most recent studies of ADG are based on SNPs or haplotype alone, and systematic association study for this complex trait based on CNVs is still missing.

In this study, we presented a comprehensive CNV association analysis for ADG in Chinese Simmental beef cattle. Seven CNVs were identified significantly associated with ADG using probed-base association analysis. Notably, we found one common deletion with $89 \mathrm{~kb}$ imbedded in LHFPL6 with high frequency and one rare deletion overlapped with GRB10 as potential candidate variants for ADG in Chinese Simmental cattle. Further systematic studies indicated the identified common deletion may contribute additional effect to ADG beyond SNPs.

\section{Results}

\section{CNV identification}

We performed CNV analysis with the Illumina Bovine HD BeadChip in Chinese Simmental beef cattle. A total of 234,973 raw $\mathrm{CNV}$ events were generated using PennCNV v1.0.4 [26] based on the UMD3.1 genome assembly. After quality control, 61,710 of them in 1079 individuals that met quality thresholds were kept for subsequent analyses. On average, 57.2 CNV events were obtained for each individual, with average length of 3.6 $\mathrm{Mb}$ (Additional file 1). These CNVs were merged into 4912 nonredundant copy number variation regions (CNVRs) with a total length of $\sim 248.7 \mathrm{Mb}$, corresponding to $\sim 8.9 \%$ of the cattle genome.

\section{Enrichment analysis using CNV-disrupting genes}

We further investigated the gene-disrupting CNVs using the DAVID (The Database for Annotation, Visualization and Integrated Discovery) system to check enrichment for these genes. Duplication and deletion were considered separately in current study. We obtained 1863 and 629 genes overlapped with deletion and duplication regions, respectively (Additional file 2). Using DAVID annotation platform, for deletions we found that a significant over-representation of genes related to antigen processing and presentation of peptide or polysaccharide antigen via MHC class II and MHC class II protein complex, while for duplications we found that several genes were enriched in MHC class I protein complex, antigen processing and presentation of peptide antigen via MHC class I, immune response, antigen processing and presentation of peptide or polysaccharide antigen via MHC class II and MHC class II protein complex (Additional file 3).

\section{CNVs overlap with QTL associated with ADG trait}

We next explored the overlap of QTLs on CNV regions (at least $1 \mathrm{bp}$ overlap between them). We retrieved autosomal QTL regions from QTLdb associated with the trait classes 'Average daily gain'. We found that 356 deletion and 135 duplication regions overlapped with the merged QTL regions for ADG. Among them, deletion regions occupy $\sim 14.13 \mathrm{Mb}$, while duplication regions occupy $\sim 4.08 \mathrm{Mb}$ (Additional file 4 ). These findings imply these CNVs is likely to be used as new potential candidate markers to refine cattle QTLs after validation.

\section{ADG associated CNVs}

We carried out probe-based CNV association analysis for ADG, and this approach converts the individual-level $\mathrm{CNV}$ calls into population-level probe-based CNV. The probe-based $\mathrm{CNV}$ table was generated by running the ParseCNV.pl script with the includePed option implemented in ParseCNV2.0 program [27]. We then conducted an association test for ADG using the mixed linear models plugged in the EMMAX software. In this study, deletion-only and duplication-only models were utilized to separately detect the associated deletion and duplication regions. We obtained 62,952 and 21,802 probes within deletion and duplication regions for subsequent association analysis, respectively. Using mixed linear models, we identified 24 and 12 significant SNP probes within deletion and duplication region based on genome-wide significant thresholds, where $P$ values were set to 1.59E-05 for deletions and 4.59E-05 for duplications as suggested by ParseCNV [27] (Additional file 5). Manhattan plots for probe-based $\mathrm{CNV}$ association analysis of deletion and duplication CNVs for average dairy gain were presented in Fig. 1a and b. 
A

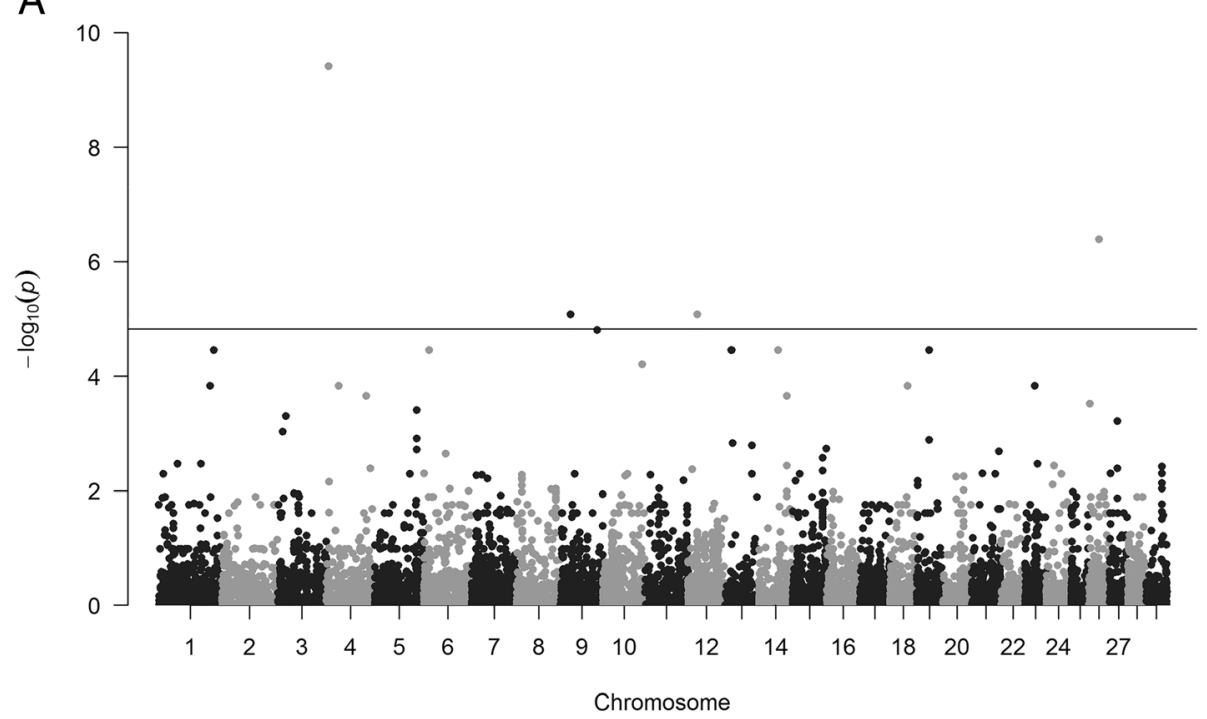

B

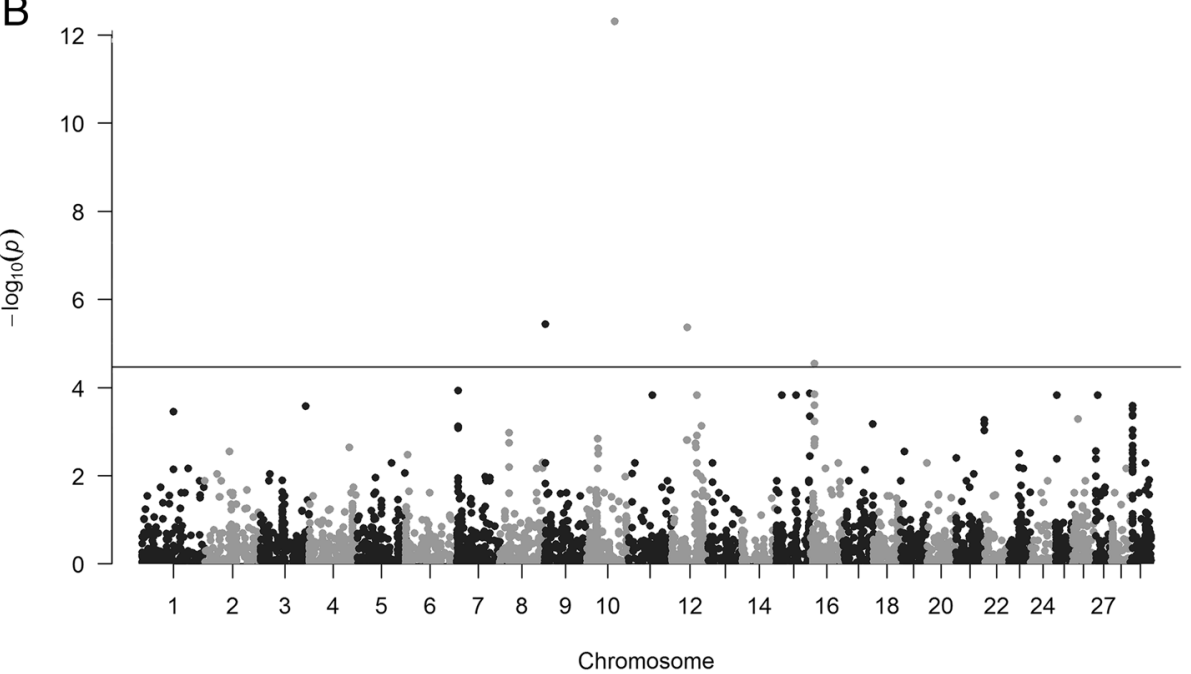

Fig. 1 Manhattan plots for probe-based CNV association analysis. a Genome wide association results of deletion CNVs for average dairy gain. b Genome wide association results of duplication CNVs for average dairy gain. The - $\log _{10}(P$ value) of each probe ( $y$-axis) in the associationanalysis using EMMMAX algorithm is plotted against the genomic position (x-axis)

Based on the significant level of probes in CNVRs, we defined the candidate regions as the CNVRs with at less two significant probes under the suggestive significant level. The 36 significant probes were detected within 5 deletion CNVRs and 2 duplication CNVRs (CNV with only one significat probe was not included), which ranged from $9265 \mathrm{bp}$ to $89,050 \mathrm{bp}$ in length (Table 1). However, we found two top probes associated with ADG using a FDR multiple-correction $(P<0.01)$. One probe with deletion located at $5.1 \mathrm{Mb}$ on BTA4 ( $\left.\mathrm{P}_{\mathrm{FDR}}=3.02 \mathrm{E}-04\right)$, and one probe within duplication located at $\sim 68.1 \mathrm{Mb}$ on BTA10 $\left(\mathrm{P}_{\mathrm{FDR}}=1.54 \mathrm{E}-04\right)$. Among the identified seven CNVRs, we identified 2 deletions imbedded with genes including LHFPL6 and SORCS3. One deletion within LHFPL6 on BTA12 shows a highest frequency of $12.9 \%$, while other deletions display relatively low frequencies and located at BTA4, BTA9 and BTA12.

Beside deletions, we also identified two candidate duplications for ADG. However, no gene was found within these duplication regions. In addition, we found one duplication with $125 \mathrm{~kb}$ displaying a frequency of $0.74 \%$ in our population. One significant probe in duplication located at the upstream of R3HDM2, but only one significant probe was detected for this duplication.

Besides the probe-based approach for $\mathrm{CNV}$ association, we also examined CNV calls affecting potential regions for $\mathrm{ADG}$ using an alternative region-based method, which have been previously described in CNVtools [28]. Using region-based association, we only observed one associated rare deletion with $9265 \mathrm{~kb}$ at 
Table 1 Candidate copy number variation regions associated with average dairy gain for beef cattle

\begin{tabular}{llllllll}
\hline CNV Type & BTA & Start & End & Length $(\mathrm{bp})$ & Count of significant probes & Distance (bp) & Candidate Genes \\
\hline Del & 4 & $5,081,669$ & $5,090,934$ & 9265 & 5 & 13,965 & GRB10 \\
Del & 9 & $25,021,405$ & $25,050,866$ & 29,461 & 10 & 212,896 & CENPW \\
Del & 9 & $90,417,787$ & $90,428,353$ & 10,566 & 3 & 161,602 & ESR1 \\
Del & 12 & $22,890,419$ & $22,979,469$ & 89,050 & 2 & within & LHFPL6 \\
Del & 26 & $25,675,473$ & $25,682,667$ & 7194 & 4 & within & SORCS3 \\
Dup & 9 & $2,688,360$ & $2,760,007$ & 71,647 & 8 & $1,972,937$ & PHF3 \\
Dup & 10 & $68,148,124$ & $68,150,780$ & 2656 & 4 & 37,825 & ATG14 \\
\hline
\end{tabular}

5.1 Mb on BTA4, which was located at $\sim 14 \mathrm{~kb}$ upstream of GRB10 (Growth Factor Receptor Bound Protein 10) (Fig. 2a and b), while no significant signal was observed among other candidate CNV regions.

In addition, to ensure reliability of our CNV detection method, we randomly selected seven identified CNVs representing different types for quantitative PCR (qPCR), and examine eight samples which contain each of seven CNVs. Two distinct pairs of primers were designed using Primer 3.0 for each detected CNV (Additional file 6). Our analysis showed that the validation rates of the eight samples varied from 71.43 to $100 \%$ with an average of $85.71 \%$, which were comparable to our earlier results and other studies [29-33].

\section{Selection estimation and sequencing validation for one common deletion}

To investigate the selection involved with CNVs, we further extracted the values of LogRR for the candidate regions in 188 Chinese native cattle. Notably, we found the $89 \mathrm{~kb}$ deletion (with 34 SNPs) located at BTA12 showed obvious difference for average LogRR between Del-carrier (i.e. Deletion carrying) individuals and Normal individuals in Chinese Simmental cattle (Fig. 3a). Using the $\mathrm{V}_{S T}$ statistics, we also obtained several peak with high $\mathrm{V}_{S T}$ values within the regions (BTA12:22-23 Mb) (Fig. 3b) for the comparison between Chinese Simmental cattle between and four groups of Chinese native cattle (North group, Northwest group, South group and Southwest group) [34]. Our result suggested this candidate $\mathrm{CNV}$ region for ADG with high frequency may be under positive selection in Chinese Simmental beef cattle.

We then extracted the whole genome sequencing reads available for four individuals with deletions as predicted by BovineHD SNP array data. Integrative Genomics Viewer (IGV, http://software.broadinstitute.org/software/ $\mathrm{igv} /$ ) was utilized to capture the changes of NGS data [35]. In all Del-carrier animals, the occurrence of deletion was obviously observed from the sequencing dataset. Notably, we found clear changes for this deletion across samples, which indicates potential copy number deletion when compared with normal samples (Fig. 4a). Next, we extracted the 34 SNPs within this region and generated the LD blocks, we observed several blocks with high LD patterns covering the right part of this deletion region
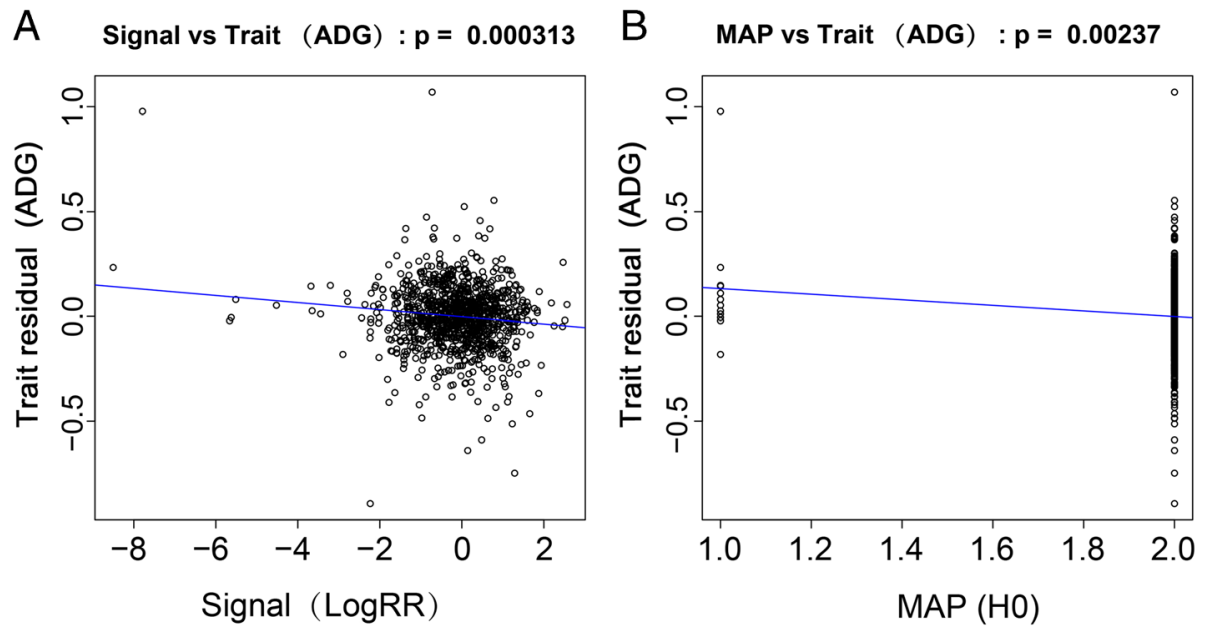

Fig. 2 Region association of ADG for CNV region at 5.1 Mb on BTA4. a The adjusted trait residuals against signal (LogRR) for CNV region at 5.1 Mb on BTA4. $\mathbf{b}$ The adjusted trait residuals against copy number state (MAP) estimated by mixture model assignment 

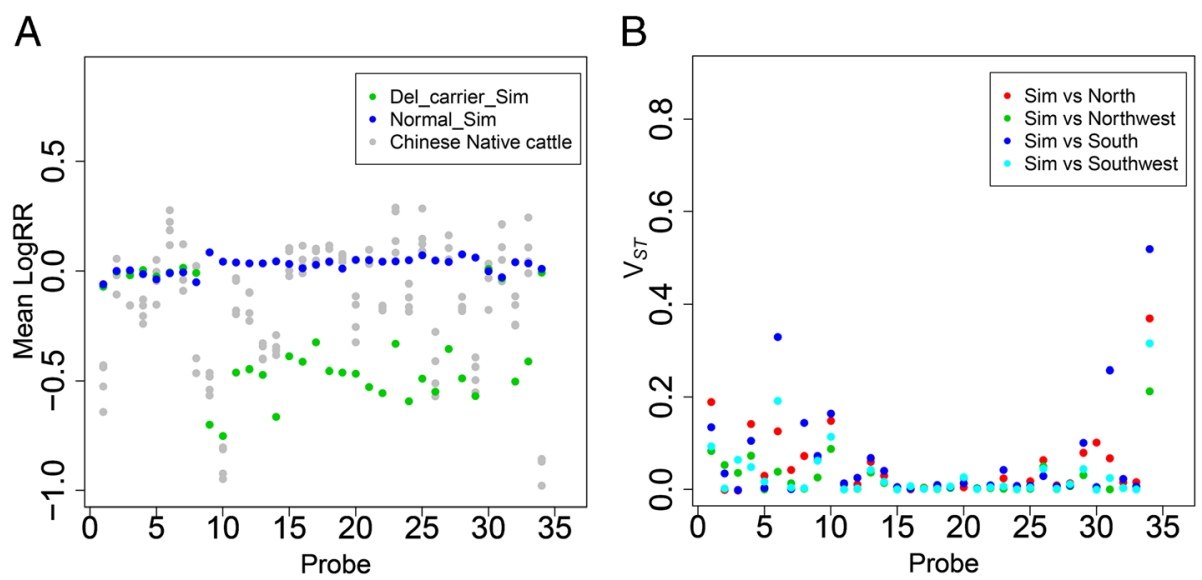

Fig. 3 a Mean LogRR plot of the CNV region at BTA12. Each point shows the mean LogRR of three groups: Simmental with CNV (Del-carrier) are colored by green, Simmental without CNV (Normal) are colored by blue, while Chinese native cattle are colored by grey. $\mathbf{b}$ Estimation of $V_{S T}$ based on LogRR for CNV regions between pairwise-groups, red points represent Simmental vs. North group, green points represent Simmental vs. Northwest group, blue color represents Simmental vs. South group, cyan points represent Simmental vs. Southwest group

(Fig. 4b). However, no candidate SNP for ADG were found from previous reports, therefore we suspected this deletion is likely be one of important structural variants that contribute to the change of ADG in beef cattle.

\section{Discussion}

Genome wide association studies have remarkably advanced our understanding of the genetic basis of complex traits. However, these strategies cannot fully evaluate the overall heritability as other genomic variants may contribute effect for these traits [1], thus elucidation of genetic mechanism of $\mathrm{CNV}$ for complex traits still needs to be further investigated $[36,37]$.

Despite the improvements in genotyping platforms and statistic approaches have facilitated the discovery of $\mathrm{CNVs}$, integrating CNVs analysis into GWAS for complex traits remains challenging. Although it is possible that CNVs are in linkage disequilibrium (LD) with associated variants, the identification of causal variants may still require us to consider CNVs beside SNPs. Previous studies of CNV association for complex trait in farm animal are mostly done using common CNVs detected by a multivariate analysis $[11,12,38]$. These approaches utilize the copy number analysis module under the multivariate option, and thus, facilitate the identification of common CNV segments. However, the CNAM algorithm force the CNV boundaries within a fixed window, which may cause CNV boundary enforcement artifacts. Compared to CNAM method, probe based association implemented in ParseCNV was developed to facilitate data processing and improve transparency for $\mathrm{CNV}$ association studies [27]. ParseCNV converts the individual level CNV calls into population level probe-based CNV states, thus this process can facilitate variable construction for association test based on $\mathrm{CNV}$.
To systematically search for CNVs that contribute genetic architectures of ADG, we conducted a genome-wide association study based on CNVs using Illumina Bovine $770 \mathrm{~K}$ BeadChip in Chinese Simmental cattle. Our previous studies identified $263 \mathrm{CNV}$ regions (CNVRs), which covering 35.48 $\mathrm{Mb}(1.41 \%)$ of the cattle genome in $\sim 700$ individuals [29]. In present study, we found $248.7 \mathrm{Mb}$, corresponding to $8.9 \%$ genome. This probably is due to larger sample size was used for $\mathrm{CNV}$ discovery in our populations. Large population can facilitate the application of CNV-based GWAS analysis and help to improve the detection of potentially associated CNV for ADG. In addition, PCR-based validation results showed around $86 \%$ of the validation based on qPCR were consistent with the PennCNV predictions. Also, CNV annotation indicates several genes with significant over-representation were related to receptor activity, immune and antigen processing, which are consistent with previous CNV analyses in cattle and other mammals [30, 39-42]. Totally, using probe-based CNV association analysis, we identified 38 significant probes and 7 corresponding $\mathrm{CNV}$ regions associated with ADG. This finding, for the first time, reported the associated CNVs contributing to ADG in farm animals. Our previous study has identified 40 significant SNPs and 7 prominent genes for ADG using multi-strategy GWAS in Chinese Simmental beef cattle [25]. Additionally, no SNPs, genes and regions in this SNP-based GWAS was found overlapped with the identified CNVs in the current study. Thus, the CNV deletions discovered in present study might contribute to ADG alone.

Totally, we have identified several candidate genes (e.g. LHFPL6, SORCS3, GRB10, CENPW, ESR1 and ATG14) within or near candidate CNVs for ADG. Among them, we found one common deletion imbedded in LHFPL6 at 22.9 Mb on BTA12 with high frequency in Chinese Simmental population. This gene belongs to a member of 

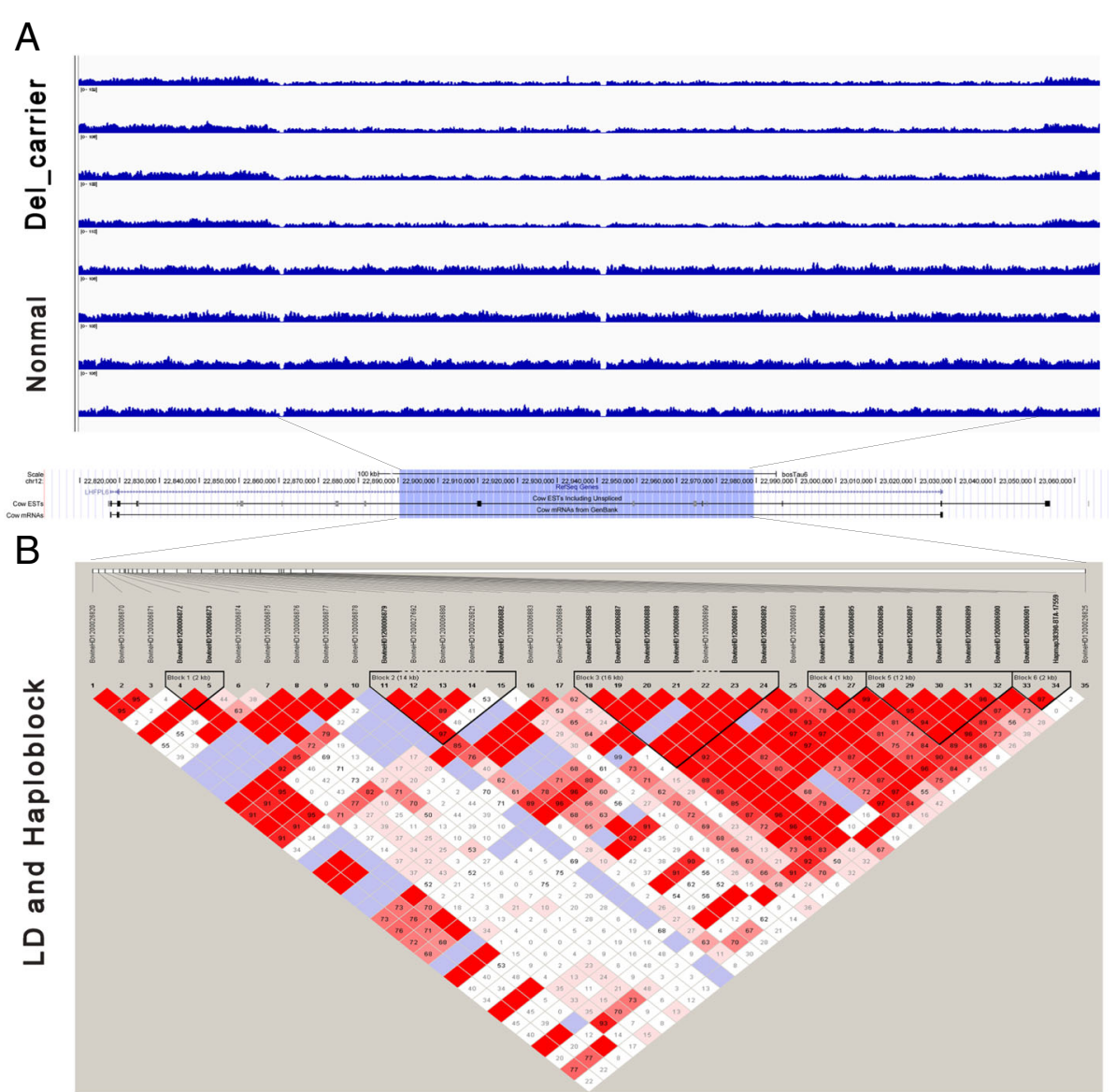

Fig. 4 a Identification of an $89 \mathrm{~kb}$ deletion affecting ADG by genome sequencing screen captures from the IGV program depicting aligned reads around BTA12: 22890419-22,979,469 bp (top panel) after mapping to the UMD3.1 reference genome. The low read depths was indicative of deletions, which was confirmed by PCR. $\mathbf{b}$ LD plot of SNPS covering the entire CNV region at BTA12. Linkage-disequilibrium pattern across the CNV region and flanking regions for the 34 SNPs. Six haplotypes are shown as predicted by Haploview

the lipoma HMGIC fusion partner (LHFP) gene family, which was reported that fused to a high-mobility group gene in a translocation-associated lipoma. Mutations in LHFP-like gene was found that related to the deafness in mice and humans [43, 44]. Moreover, we suspected the high frequency deletion occurred under positive selection and may play an important role to affect complex traits. Also, our $V_{S T}$ statistic results suggested this deletion display significant association with ADG in Chinese Simmental cattle compared to native cattle. Therefore, this CNV may potentially act as important genome variant under selection contributing to ADG.

In addition, we identified one rare deletion near GRB10 located at $5.1 \mathrm{Mb}$ on BTA4 using both probe-based and region based association analyses. GRB10, growth factor receptor-bound protein 10 gene, is an intracellular adaptor protein that acts as a negative regulator of insulin and insulin-like growth factor receptors to restrict fetal and placental growth during mammalian development $[45,46]$. This gene have been identified as candidate imprinted gene associated with growth-related trait in Irish Holstein-Friesian cattle [47, 48]. GRB10 has also been reported to be related to the development of fiber number in skeletal muscle [49] and milk tridecylic acid [50]. However, the functional study of these identified deletions still need more efforts to be further explored with third generation sequencing and other experimental validations. Our analyses provided some valuable insights into the understanding the missing heritability of ADG. To our knowledge, the present study provides the first case of association between CNVs and quantitative trait in Chinese Simental beef cattle. These results extend our understanding of $\mathrm{CNV}$ in complex trait and pinpoint to the importance of utilizing new methods that allow for considering these variations in genome-wide association [51]. Further functional study and expression assays can be utilized to assess the biological effects of CNVs in candidate genes and help to understand their contribution to complex traits in farm animals. 


\section{Conclusions}

Our study identified 24 and 12 significant SNP probes within four deletions and three duplications for ADG, respectively. Among them, we found one common CNV deletion with $89 \mathrm{~kb}$ imbedded in LHFPL6 at $22.9 \mathrm{Mb}$ on BTA12, this deletion was not overlapped with any candidate SNP for ADG compared with previous SNPs-based association studies, suggesting its important role for ADG. In addition, we identified one rare deletion near GRB10 at 5.1 Mb on BTA4 for ADG using both probe-based association and region-based approaches. Our results provided some valuable insights to elucidate the genetic basis of ADG in beef cattle, these findings offer an alternative perspective to understand the genetic mechanism of complex traits in terms of copy number variations in farm animals.

\section{Methods}

\section{Ethics statement}

No ethics statement was required for the collection of genetic material. The data from animals included in this study were derived from previous analyses that obtained specific permissions [25].

\section{Samples and phenotype data}

Samples were genotyped using Illumina Bovine HD SNPs array. A more detailed description of the original array data set can be found in our previous publication [25]. The resource population consisted of 1173 Simmental cattle that were born between 2008 and 2013 in Ulgai, Inner Mongolia. After weaning, all calves were transferred to a fattening farm in Beijing and fattened in the same pens for 8 12 months. All animals were fed with same feeding and management conditions, and ADG was estimated during the fattening period. Test distribution of ADG trait showed it follow a normal distribution and analysis of variance (ANOVA) showed that farm, sex, year of measurement, fattening days had significant effects $(P<0.01)$. Thus, these factors were adjusted in the linear regression model, and the resulting trait residual was further considered for ADG association test.

\section{CNVs detection}

PennCNV v1.0.4 software was utilized to identify CNV across autosomes [26]. PennCNV incorporates both the Log $\mathrm{R}$ Ratio (LogRR) value and the frequency of allele B (BAF) for $\mathrm{CNV}$ detection. The CNV calling was carried out following the previous study by Yang et al. [34]. The final CNV events were produced by keeping high quality samples according to the following criteria: call rate $>0.95$, standard deviation (SD) of $\mathrm{LRR}<0.35$, and $\mathrm{GC}$ waviness factors as 0.005 .

\section{CNV association analysis}

To identify CNV regions associated with ADG, CNV calls and quality measures were translated to probe level using ParseCNV [27]. ParseCNV proposes an integrative $\mathrm{CNV}$ association method that convert $\mathrm{CNV}$ calls into probe-based statistics for individual CNVs. As CNV boundaries vary across individuals, the beginning and end points of CNVs may be unclear, we are not able to classify different CNVs as identical or different, thus $\mathrm{CNV}$ association test were performed at the probe level.

We tested the frequency of SNP probes affected by various $\mathrm{CNV}$ types separately, i.e. deletions, duplications and genomic regions affected by both types of CNV. The association between CNV carrier frequencies and ADG across population were evaluated using linear mixed model implemented in EMMAX software [52]. Relatedness among individuals was utilized as random effects based on SNPs genotype. For CNV association, a suggestive genome wide threshold was considered in present study as suggested by [27]. The probe-based statistical significance ( $\log _{10} P$-value) of neighboring probes were calculated using EMMAX method. Then the neighboring SNPs with comparable significance were collapsed into CNVRs which constitute genomic span of consecutive probes (at less two probes). The local lowest $P$-value for identified probes was used to represent the significant level of association of CNVR. Accordingly, a multiple correction was carried out for each probe using qvalue package [53], and $q$ value $<0.05$ was used to determine level of significance.

\section{Region-based CNV association analyses}

We next utilized the density of probes within $\mathrm{CNV}$ regions to assess the possible enrichment of region-based CNVs. The cumulative burden of CNVs can be effectively estimated on a region level using the approach implemented in CNVtools [28]. It combines the information across $\mathrm{CNV}$ probes to obtain a one-dimensional signal using principal component and Bayesian information criterion for each sample. A copy number genotype was assigned to each locus for each individual to test for genetic association with a quantitative trait based on a standard regression approach. The exact boundaries of the candidate regions were based on the BosTau6 (UMD 3.1) reference assembly.

\section{Pathway analysis and CNV genes annotation}

We searched the genes affected by the identified CNVs using UCSC genome browser (UMD 3.1). Any refSeq genes that was either fully included or broken by $\mathrm{CNV}$ that were considered as CNV affected. To evaluate the effects of disrupted genes from any particular functionally defined molecular pathway, we investigated the CNV-disrupting genes using the DAVID gene functional classification system [54]. Deletion and duplication were considered separately. To avoid false positives, we further considered that enriched pathway which have at 
least two genes and the $P$ value $<0.05$ after the Bonferroni correction for multiple testing.

\section{CNVs overlapped with QTLs associated with ADG traits} QTLs information were downloaded from cattle QTLdb [55]. We merged all QTL regions into a set of unique non-redundant regions. The coordinates of QTLs based on Btau_4.0 were converted to UMD3.1. The liftOver conversion between assemblies was conducted at a relaxed threshold (Minimum ratio of bases that must remapped was set to $75 \%$ ).

\section{Next generation sequencing analysis}

Genomic DNA from four Chinese Simmental bulls was extracted from blood samples using a TIANamp Blood DNA Kit (Tiangen Biotech Company limited, Beijing, China), and DNA with an A260/280 ratio between 1.8 and 2.0 were subjected to further library construction. Two paired-end libraries were constructed for each individual, the read length was $2 \times 150 \mathrm{bp}$, and whole genome sequencing was performed using Illumina Hiseq2500 instruments (Illumina Inc., San Diego, CA, USA). All processes were performed according to the standard manufacturer's protocols. Each sample was sequenced to an approximate coverage of $20 \mathrm{X}$. We removed low-quality reads following filters: (1) reads with an adaptor, (2) reads containing more than $10 \%$ unknown bases, (3) reads containing more than 50\% low-quality bases. After filtering, we used the bwa-0.7.8 with parameters (mem -t $4-\mathrm{k} 32-\mathrm{M}$ ) to perform sequence alignment based on the UMD3.1 genome assembly [56].

\section{Quantitative PCR validation}

Quantitative PCR (qPCR) was utilized to validate seven associated CNVs detected by PennCNV. For each CNV, primers were designed using Primer3 web tool (http:// bioinfo.ut.ee/primer3-0.4.0/primer3/). To ensure the amplification efficiencies, standard curve of each pair of primer was generated using template from serial diluted genomic DNA sample of a common cattle. The Basic Transcription Factor 3 (BTF3) gene was selected as the control assuming two copies of DNA segment. With a total volume of $20 \mu \mathrm{L}$ reagents in a 96-well plate, qPCR was conducted using SYBR green chemistry in triplicate reactions on ABI STEPONE plus, thermo Real-Time PCR System. The condition for thermal cycle was as follows: 2 min at $95^{\circ} \mathrm{C}$ followed by 40 cycles at $95^{\circ} \mathrm{C}$ for 10 $\mathrm{s}, 60^{\circ} \mathrm{C}$ for $40 \mathrm{~s}$. We calculated the relative copy number for each selected region using the $2^{-\Delta \Delta C T}$ method. First, the average CT value of three replications of each sample and normalized against the control gene, then $\triangle \mathrm{CT}$ value was estimated between the $\mathrm{CNV}$ carrier sample and a reference sample with normal status.

\section{Additional files}

Additional file 1: Summary of identified CNV and CNVRs using PennCNV in Chinese Simmental beef cattle. (XLSX 3459 kb)

Additional file 2: Gene annotation of duplications and deletions for overlapped genes, CDSs and exons. (XLSX $309 \mathrm{~kb}$ )

Additional file 3: Gene ontology (GO) enrichment using DAVID for CNVs. (XLSX $233 \mathrm{~kb})$

Additional file 4: Deletion and duplication regions overlapped with the merged QTL regions for ADG. (XLSX $37 \mathrm{~kb}$ )

Additional file 5: Summary of probe-based CNV association analysis results including probe name, chromosome, position, $P$-value, and adjusted q values. (XLSX $3735 \mathrm{~kb}$ )

Additional file 6: Primers information and $\mathrm{qPCR}$ validations of seven CNVs. (XLSX $11 \mathrm{~kb})$

\section{Abbreviations}

ADG: Average daily gain; CNVR: Copy number variation region;

BAF: Frequency of allele B; BTA: Bos Taurus autosomes; CNV: Copy number variation; GWAS: Genome-wide association study; IVG: Integrative genomics viewer; LogRR: Log R ratio; QTL: Quantitative trait locus; SNP: Single nucleotide polymorphism

\section{Acknowledgements}

The authors would like to thank the staffs at the cattle experimental unit in Beijing and Ulgai for caring of animals and collection biological samples.

\section{Funding}

This study was supported by the National Natural Science Foundation of China (31702084) and Agricultural Science and Technology Innovation Program of China (ASTIP-IAS-TS-9, ASTIP-IAS-03 and ASTIP-IAS-TS-16) for the design of the study and sample collection. Also, this study was supported by the Elite Youth Program in Chinese Academy of Agricultural Sciences for the data analysis and interpretation of the study.

Availability of data and materials

Datasets are available from the Dryad Digital Repository (doi: https://doi.org/ 10.5061/dryad.4qc06).

Consent to participate

Not applicable.

\section{Authors' contributions}

Conceived and designed the experiments: LYX, GEL and JYL. Performed the experiments: LW, LPZ and XG. Analyzed the data: LY, HJG and BZ. Contributed reagents/materials/analysis tools: $L Y, Y C$ and JYL. Wrote the paper: LYX, GEL and $\mathrm{JYL}$. All authors have read and approved the manuscript.

\section{Ethics approval and consent to participate}

No ethics statement was required for the collection of genetic material. The data from animals included in this study were derived from previous analyses that obtained specific permissions [25].

Consent for publication

Not applicable.

\section{Competing interests}

The authors declare that they have no competing interests except that George Liu is a member of the editorial board (Associate Editor) of this journal.

\section{Publisher's Note}

Springer Nature remains neutral with regard to jurisdictional claims in published maps and institutional affiliations.

\section{Author details}

${ }^{1}$ Innovation Team of Cattle Genetic Breeding, Institute of Animal Sciences, Chinese Academy of Agricultural Sciences, Beijing 100193, China. ${ }^{2}$ Farm 
Animal Genetic Resources Exploration and Innovation Key Laboratory of Sichuan Province, Sichuan Agricultural University, Chengdu 611130, China. ${ }^{3}$ Beijing Genecast Biotechnology Co., Beijing 100191, China. ${ }^{4}$ U.S. Department of Agriculture-Agricultural Research Services, Animal Genomics and Improvement Laboratory, Beltsville, MD 20705, USA.

Received: 18 July 2018 Accepted: 20 December 2018

Published online: 10 January 2019

\section{References}

1. Scherer SW, Lee C, Birney E, Altshuler DM, Eichler EE, Carter NP, Hurles ME, Feuk L. Challenges and standards in integrating surveys of structural variation. Nat Genet. 2007;39(7 Suppl):S7-15.

2. Zhang F, Gu W, Hurles ME, Lupski JR. Copy number variation in human health, disease, and evolution. Annu Rev Genomics Hum Genet. 2009;10: 451-81.

3. Henrichsen CN, Vinckenbosch N, Zollner S, Chaignat E, Pradervand S, Schutz F, Ruedi M, Kaessmann H, Reymond A. Segmental copy number variation shapes tissue transcriptomes. Nat Genet. 2009;41(4):424-9.

4. Stranger BE, Forrest MS, Dunning M, Ingle CE, Beazley C, Thorne N, Redon R, Bird CP, de Grassi A, Lee C, et al. Relative impact of nucleotide and copy number variation on gene expression phenotypes. Science. 2007;315(5813): 848-53.

5. Gamazon ER, Nicolae DL, Cox NJ. A study of CNVs as trait-associated polymorphisms and as expression quantitative trait loci. PLoS Genet. 2011; 7(2):e1001292.

6. Estivill X, Armengol L. Copy number variants and common disorders: filling the gaps and exploring complexity in genome-wide association studies. PLoS Genet. 2007;3(10):1787-99.

7. Zahnleiter D, Uebe S, Ekici AB, Hoyer J, Wiesener A, Wieczorek D, Kunstmann E, Reis A, Doerr HG, Rauch A, et al. Rare copy number variants are a common cause of short stature. PLoS Genet. 2013;9(3):e1003365.

8. Mace A, Tuke MA, Deelen P, Kristiansson K, Mattsson H, Noukas M, Sapkota $Y$, Schick U, Porcu E, Rueger S, et al. CNV-association meta-analysis in 191,161 European adults reveals new loci associated with anthropometric traits. Nat Commun. 2017;8(1):744

9. Willer CJ, Speliotes EK, Loos RJ, Li S, Lindgren CM, Heid IM, Berndt SI, Elliott $A L$, Jackson AU, Lamina $C$, et al. Six new loci associated with body mass index highlight a neuronal influence on body weight regulation. Nat Genet. 2009;41(1):25-34

10. Hou Y, Liu GE, Bickhart DM, Matukumalli LK, Li C, Song J, Gasbarre LC, Van Tassell CP, Sonstegard TS. Genomic regions showing copy number variations associate with resistance or susceptibility to gastrointestinal nematodes in Angus cattle. Funct Integr Genomics. 2012;12(1):81-92.

11. Xu L, Hou Y, Bickhart DM, Song J, Van Tassell CP, Sonstegard TS, Liu GE. A genome-wide survey reveals a deletion polymorphism associated with resistance to gastrointestinal nematodes in Angus cattle. Funct Integr Genomics. 2014:14(2):333-9.

12. Xu L, Cole JB, Bickhart DM, Hou Y, Song J, VanRaden PM, Sonstegard TS, Van Tassell CP, Liu GE. Genome wide CNV analysis reveals additional variants associated with milk production traits in Holsteins. BMC Genomics. 2014:15:683.

13. Hou Y, Bickhart DM, Chung H, Hutchison JL, Norman HD, Connor EE, Liu GE. Analysis of copy number variations in Holstein cows identify potential mechanisms contributing to differences in residual feed intake. Funct Integr Genomics. 2012;12(4):717-23

14. Glick G, Shirak A, Seroussi E, Zeron Y, Ezra E, Weller JI, Ron M. Fine mapping of a QTL for fertility on BTA7 and its association with a CNV in the Israeli Holsteins. G3. 2011:1(1):65-74.

15. Kadri NK, Sahana G, Charlier C, Iso-Touru T, Guldbrandtsen B, Karim L, Nielsen US, Panitz F, Aamand GP, Schulman N, et al. A 660-Kb deletion with antagonistic effects on fertility and milk production segregates at high frequency in Nordic red cattle: additional evidence for the common occurrence of balancing selection in livestock. PLoS Genet. 2014;10(1):e1004049.

16. Lindholm-Perry AK, Kuehn LA, Oliver WT, Sexten AK, Miles JR, Rempel LA, Cushman RA, Freetly HC. Adipose and muscle tissue gene expression of two genes (NCAPG and LCORL) located in a chromosomal region associated with cattle feed intake and gain. PLoS One. 2013;8(11):e80882.

17. Hoshiba H, Setoguchi K, Watanabe T, Kinoshita A, Mizoshita K, Sugimoto Y, Takasuga A. Comparison of the effects explained by variations in the bovine PLAG1 and NCAPG genes on daily body weight gain, linear skeletal measurements and carcass traits in Japanese black steers from a progeny testing program. Anim Sci J. 2013;84(7):529-34.

18. Peters SO, Kizilkaya K, Garrick DJ, Fernando RL, Reecy JM, Weaber RL, Silver GA, Thomas MG. Bayesian genome-wide association analysis of growth and yearling ultrasound measures of carcass traits in Brangus heifers. J Anim Sci. 2012:90(10):3398-409.

19. Rolf MM, Taylor JF, Schnabel RD, McKay SD, McClure MC, Northcutt SL, Kerley MS, Weaber RL. Genome-wide association analysis for feed efficiency in Angus cattle. Anim Genet. 2012;43(4):367-74.

20. Lu D, Miller S, Sargolzaei M, Kelly M, Vander Voort G, Caldwell T, Wang Z, Plastow G, Moore S. Genome-wide association analyses for growth and feed efficiency traits in beef cattle. J Anim Sci. 2013;91(8):3612-33.

21. Lindholm-Perny AK, Kuehn LA, Oliver WT, Kern RJ, Cushman RA, Miles JR, McNeel AK, Freetly HC. DNA polymorphisms and transcript abundance of PRKAG2 and phosphorylated AMP-activated protein kinase in the rumen are associated with gain and feed intake in beef steers. Anim Genet. 2014;45(4):461-72.

22. Lindholm-Perry AK, Sexten AK, Kuehn LA, Smith TP, King DA, Shackelford $\mathrm{SD}$, Wheeler TL, Ferrell CL, Jenkins TG, Snelling WM, et al. Association, effects and validation of polymorphisms within the NCAPG - LCORL locus located on BTA6 with feed intake, gain, meat and carcass traits in beef cattle. BMC Genet. 2011:12:103.

23. Serao NV, Gonzalez-Pena D, Beever JE, Bollero GA, Southey BR, Faulkner DB, Rodriguez-Zas SL. Bivariate genome-wide association analysis of the growth and intake components of feed efficiency. PLoS One. 2013;8(10):e78530.

24. Lindholm-Perry AK, Kuehn LA, Snelling WM, Smith TP, Ferrell CL, Jenkins TG, King DA, Shackelford SD, Wheeler TL, Freetly HC. Genetic markers on BTA14 predictive for residual feed intake in beef steers and their effects on carcass and meat quality traits. Anim Genet. 2012;43(5):599-603.

25. Zhang W, Li J, Guo Y, Zhang L, Xu L, Gao X, Zhu B, Gao H, Ni H, Chen Y. Multistrategy genome-wide association studies identify the DCAF16-NCAPG region as a susceptibility locus for average daily gain in cattle. Sci Rep. 2016;6:38073.

26. Wang K, Li M, Hadley D, Liu R, Glessner J, Grant SF, Hakonarson H, Bucan M. PennCNV: an integrated hidden Markov model designed for high-resolution copy number variation detection in whole-genome SNP genotyping data. Genome Res. 2007;17(11):1665-74.

27. Glessner JT, Li J, Hakonarson H. ParseCNV integrative copy number variation association software with quality tracking. Nucleic Acids Res. 2013;41(5):e64.

28. Barnes C, Plagnol V, Fitzgerald T, Redon R, Marchini J, Clayton D, Hurles ME. A robust statistical method for case-control association testing with copy number variation. Nat Genet. 2008;40(10):1245-52.

29. Wu Y, Fan H, Jing S, Xia J, Chen Y, Zhang L, Gao X, Li J, Gao H, Ren H. A genome-wide scan for copy number variations using high-density single nucleotide polymorphism array in Simmental cattle. Anim Genet. 2015;46(3): 289-98.

30. Hou Y, Liu GE, Bickhart DM, Cardone MF, Wang K, Kim ES, Matukumalli LK, Ventura M, Song J, VanRaden PM, et al. Genomic characteristics of cattle copy number variations. BMC Genomics. 2011;12:127.

31. Gao Y, Jiang J, Yang S, Hou Y, Liu GE, Zhang S, Zhang Q, Sun D. CNV discovery for milk composition traits in dairy cattle using whole genome resequencing. BMC Genomics. 2017;18(1):265.

32. Choi JW, Lee KT, Liao X, Stothard P, An HS, Ahn S, Lee S, Lee SY, Moore SS, Kim TH. Genome-wide copy number variation in Hanwoo, Black Angus, and Holstein cattle. Mamm Genome. 2013:24(3-4):151-63.

33. Sasaki S, Watanabe T, Nishimura S, Sugimoto Y. Genome-wide identification of copy number variation using high-density single-nucleotide polymorphism array in Japanese black cattle. BMC Genet. 2016;17:26.

34. Yang L, Xu L, Zhu B, Niu H, Zhang W, Miao J, Shi X, Zhang M, Chen Y, Zhang L, et al. Genome-wide analysis reveals differential selection involved with copy number variation in diverse Chinese cattle. Sci Rep. 2017;7(1):14299.

35. Robinson JT, Thorvaldsdottir H, Winckler W, Guttman M, Lander ES, Getz G, Mesirov JP. Integrative genomics viewer. Nat Biotechnol. 2011;29(1):24-6.

36. Manolio TA, Collins FS, Cox NJ, Goldstein DB, Hindorff LA, Hunter DJ, McCarthy MI, Ramos EM, Cardon LR, Chakravarti A, et al. Finding the missing heritability of complex diseases. Nature. 2009:461(7265):747-53.

37. Eichler EE, Flint J, Gibson G, Kong A, Leal SM, Moore JH, Nadeau JH. Missing heritability and strategies for finding the underlying causes of complex disease. Nat Rev Genet. 2010;11(6):446-50.

38. Zhou Y, Utsunomiya YT, Xu L, Hay el HA, Bickhart DM, Alexandre PA, Rosen BD, Schroeder SG, Carvalheiro R, de Rezende Neves HH, et al. Genome-wide CNV analysis reveals variants associated with growth traits in Bos indicus. BMC Genomics. 2016;17:419. 
39. Chen C, Qiao R, Wei R, Guo Y, Ai H, Ma J, Ren J, Huang L. A comprehensive survey of copy number variation in 18 diverse pig populations and identification of candidate copy number variable genes associated with complex traits. BMC Genomics. 2012;13:733.

40. Guryev V, Saar K, Adamovic T, Verheul M, van Heesch SA, Cook S, Pravenec M, Aitman T, Jacob H, Shull JD, et al. Distribution and functional impact of DNA copy number variation in the rat. Nat Genet. 2008;40(5):538-45.

41. Sebat J, Lakshmi B, Troge J, Alexander J, Young J, Lundin P, Maner S, Massa $\mathrm{H}$, Walker M, Chi M, et al. Large-scale copy number polymorphism in the human genome. Science. 2004;305(5683):525-8.

42. Berglund J, Nevalainen EM, Molin AM, Perloski M, Consortium L, Andre C, Zody MC, Sharpe T, Hitte C, Lindblad-Toh K, et al. Novel origins of copy number variation in the dog genome. Genome Biol. 2012;13(8):R73.

43. Longo-Guess CM, Gagnon LH, Cook SA, Wu J, Zheng QY, Johnson KR. A missense mutation in the previously undescribed gene Tmhs underlies deafness in hurry-scurry (hscy) mice. Proc Natl Acad Sci U S A. 2005;102(22): 7894-9.

44. Petit MM, Schoenmakers EF, Huysmans C, Geurts JM, Mandahl N, Van de Ven WJ. LHFP, a novel translocation partner gene of HMGIC in a lipoma, is a member of a new family of LHFP-like genes. Genomics. 1999;57(3):438-41.

45. Liu F, Roth RA. Grb-IR: a SH2-domain-containing protein that binds to the insulin receptor and inhibits its function. Proc Natl Acad Sci U S A. 1995; 92(22):10287-91.

46. Charalambous M, Cowley M, Geoghegan F, Smith FM, Radford EJ, Marlow BP, Graham CF, Hurst LD, Ward A. Maternally-inherited Grb10 reduces placental size and efficiency. Dev Biol. 2010;337(1):1-8.

47. Magee DA, Sikora KM, Berkowicz EW, Berry DP, Howard DJ, Mullen MP, Evans RD, Spillane C, MacHugh DE. DNA sequence polymorphisms in a panel of eight candidate bovine imprinted genes and their association with performance traits in Irish Holstein-Friesian cattle. BMC Genet. 2010;11:93.

48. Imumorin IG, Kim EH, Lee YM, De Koning DJ, van Arendonk JA, De Donato M, Taylor JF, Kim JJ. Genome scan for parent-of-origin QTL effects on bovine growth and carcass traits. Front Genet. 2011;2:44.

49. Holt LJ, Turner N, Mokbel N, Trefely S, Kanzleiter T, Kaplan W, Ormandy CJ, Daly RJ, Cooney GJ. Grb10 regulates the development of fiber number in skeletal muscle. FASEB J. 2012;26(9):3658-69.

50. Ibeagha-Awemu EM, Peters SO, Akwanji KA, Imumorin IG, Zhao X. High density genome wide genotyping-by-sequencing and association identifies common and low frequency SNPs, and novel candidate genes influencing cow milk traits. Sci Rep. 2016;6:31109.

51. Hay EHA, Utsunomiya YT, Xu L, Zhou Y, Neves HHR, Carvalheiro R, Bickhart DM, Ma L, Garcia JF, Liu GE. Genomic predictions combining SNP markers and copy number variations in Nellore cattle. BMC Genomics. 2018;19(1):441.

52. Kang HM, Sul JH, Service SK, Zaitlen NA, Kong SY, Freimer NB, Sabatti C, Eskin E. Variance component model to account for sample structure in genome-wide association studies. Nat Genet. 2010;42(4):348-54.

53. Storey JD, Tibshirani R. Statistical significance for genomewide studies. Proc Natl Acad Sci U S A. 2003;100(16):9440-5.

54. Huang DW, Sherman BT, Lempicki RA. Systematic and integrative analysis of large gene lists using DAVID bioinformatics resources. Nat Protoc. 2008;4:44.

55. Hu ZL, Park CA, Wu XL, Reecy JM. Animal QTLdb: an improved database tool for livestock animal QTL/association data dissemination in the postgenome era. Nucleic Acids Res. 2013;41(Database issue):D871-9.

56. Li H, Durbin R. Fast and accurate short read alignment with burrowsWheeler transform. Bioinformatics. 2009;25(14):1754-60.

\section{Ready to submit your research? Choose BMC and benefit from:}

- fast, convenient online submission

- thorough peer review by experienced researchers in your field

- rapid publication on acceptance

- support for research data, including large and complex data types

- gold Open Access which fosters wider collaboration and increased citations

- maximum visibility for your research: over $100 \mathrm{M}$ website views per year

At BMC, research is always in progress.

Learn more biomedcentral.com/submissions 